\title{
Multiple Bifurcations and Chaos in a Discrete Prey-Predator System with Generalized Holling III Functional Response
}

\author{
Xia Liu, ${ }^{1}$ Yanwei Liu, ${ }^{2}$ and Qiaoping $\mathrm{Li}^{3}$ \\ ${ }^{1}$ College of Mathematics and Information Science, Henan Normal University, Xinxiang 453007, China \\ ${ }^{2}$ Institute of Systems Biology, Shanghai University, Shanghai 200444, China \\ ${ }^{3}$ School of Mathematical Sciences, Henan Institute of Science and Technology, Xinxiang 453003, China \\ Correspondence should be addressed to Xia Liu; liuxiapost@163.com
}

Received 11 August 2014; Accepted 29 October 2014

Academic Editor: Mustafa Kulenović

Copyright (C) 2015 Xia Liu et al. This is an open access article distributed under the Creative Commons Attribution License, which permits unrestricted use, distribution, and reproduction in any medium, provided the original work is properly cited.

A prey-predator system with the strong Allee effect and generalized Holling type III functional response is presented and discretized. It is shown that the combined influences of Allee effect and step size have an important effect on the dynamics of the system. The existences of Flip and Neimark-Sacker bifurcations and strange attractors and chaotic bands are investigated by using the center manifold theorem and bifurcation theory and some numerical methods.

\section{Introduction}

Lotka-Volterra model as a paradigm of the fundamental population models has been developed and widely applied in ecological sciences and other fields. Some linear and nonlinear functional responses are employed to describe the phenomena of predation including the Beddington-DeAngelis type $[1,2]$, Crowley-Martin type [3], and Holling types [4] that are more realistic and proper for modeling most prey-predator interactions [1-8]. Recently, a modified prey-predator model with a generalized Holling response function of type III was addressed [8]. The system exhibits more richer dynamics including the Hopf bifurcation of codimensions 1 and 2, Bogdanov-Takens bifurcation of codimensions 2 and 3 .

The researches of the Allee effect on biological population [9-23] imply that the Allee effect probably is a nonignorable factor in ecology, especially for the situation when population density (or size) is low (or small). That fitness of an individual in a small or sparse population decreases as the population density declines leads to Allee effect occurrence. Two most common continuous growth equations to express Allee effect for a single species are given [15-18]

$$
\dot{x}=r x\left(1-\frac{x}{K}\right)(x-m), \quad \dot{x}=r\left(1-\frac{x}{K}-\frac{l}{x+b}\right) x,
$$

which are called multiplicative Allee effect model $[15,16]$ and the additive Allee effect model $[17,18]$, respectively. Parameters $m, l$, and $b>0$ are the Allee thresholds, where $l x /(x+b)$ is termed as the strong Allee effect item if $l>b r$ and weak Allee effect item if $l<b r$. Mathematically, the systems subjected to the Allee effect can be depicted by the bistability switch, which may lead to more complex dynamics. Thus, the consideration of prey-predator model subjected to an Allee effect is more realistic and has attracted renewed interest, which motivates us to make a modification to the system presented in [8] for further study:

$$
\begin{aligned}
& \dot{x}=x\left(r\left(1-\frac{x}{K}\right)(x-m)-\frac{\beta x y}{a_{0} x^{2}+b_{0} x+1}\right), \\
& \dot{y}=y\left(-d_{0}+\frac{c_{0} x^{2}}{a_{0} x^{2}+b_{0} x+1}\right),
\end{aligned}
$$

where ${ }^{\cdot}=d / d t . x$ and $y$ denote the prey and predator densities at time $t$, respectively. $r, K, m, \beta, a_{0}, c_{0}$, and $d_{0}$ are positive constants. $b_{0}$ is positive or negative. In this paper, we will focus on the case $b_{0}>0$. In particular, $r$ and $K$ represent the intrinsic growth rate and carrying capacity of prey in the absence of predation, respectively. $m>0$ is so called the strong Allee threshold. The predator consumes the prey with functional response $\beta x^{2} y /\left(a_{0} x^{2}+b_{0} x+1\right)$, known 
as Holling type III response and contributes to its growth rate $c_{0} x^{2} y /\left(a_{0} x^{2}+b_{0} x+1\right) \cdot d_{0}$ is the maximum death rate in the absence of prey. For simplicity, take nondimensional transformations $x=K X, y=Y / \beta, s=K t$ to system (2) and rewrite $X, Y, s$ as $x, y, t$; we have

$$
\begin{aligned}
& \dot{x}=x\left(r(1-x)(x-A)-\frac{x y}{a x^{2}+b x+1}\right), \\
& \dot{y}=y\left(-d+\frac{c x^{2}}{a x^{2}+b x+1}\right),
\end{aligned}
$$

where $A=m / K, a=a_{0} K^{2}, b=b_{0} K, d=d_{0} / K$, and $c=c_{0} K$.

Many studies of discrete-time models [19-38] were made and suggested that discrete-time models described by the difference equations are more appropriate than the continuous time models when populations have nonoverlapping generations or the number of populations is small. Moreover, dynamical patterns produced in discrete-time models are much richer than those observed in continuous time models.

Applying the forward Euler scheme, we obtain the discrete-time form of system (3) as follows:

$$
\begin{aligned}
x_{k+1} & =x_{k}+\theta x_{k}\left(r\left(1-x_{k}\right)\left(x_{k}-A\right)-\frac{x_{k} y_{k}}{a x_{k}^{2}+b x_{k}+1}\right) \\
& \triangleq f\left(x_{k}, y_{k}\right), \\
y_{k+1} & =y_{k}+\theta y_{k}\left(-d+\frac{c x_{k}^{2}}{a x_{k}^{2}+b x_{k}+1}\right) \triangleq g\left(x_{k}, y_{k}\right),
\end{aligned}
$$

where $\theta>0$ is the step size.

The paper is organized as follows: in Section 2 we show the existence and stability of fixed points of (4). In Section 3 we investigate the existence of flip bifurcation and the Neimark-Sacker bifurcation. In Section 4 we show some numerical simulations to illustrate our main results. Finally, a brief discussion of (4) is given to sum up our analysis.

\section{Existence and Stability of Fixed Points}

To obtain the fixed points of system (4), it is sufficient to solve the following algebraic equations:

$$
\begin{gathered}
x\left(r(1-x)(x-A)-\frac{x y}{a x^{2}+b x+1}\right)=0, \\
y\left(-d+\frac{c x^{2}}{a x^{2}+b x+1}\right)=0 .
\end{gathered}
$$

After calculating, we have the following statement.

Lemma 1. System (4) always has the trivial fixed point $E_{0}=$ $(0,0)$ and the boundary fixed points $E_{1}=(1,0), E_{2}=(A, 0)$. If $d(a+b+1)<c<d\left(a+b / A+1 / A^{2}\right)$, (4) has a unique positive fixed point $E_{*}=\left(x_{*}, y_{*}\right)$, where

$$
\begin{aligned}
& x_{*}=\frac{b d+\sqrt{b^{2} d^{2}+4 d(c-a d)}}{2(c-a d)}, \\
& y_{*}=\frac{r\left(1-x_{*}\right)\left(x_{*}-A\right)\left(a x_{*}^{2}+b x_{*}+1\right)}{x_{*}} .
\end{aligned}
$$

To analyze dynamical properties of (4), we need to compute the Jacobian matrix of (4) at any fixed point $E=$ $(x, y)$

$$
M_{E}=\left(\begin{array}{ll}
f_{x} & f_{y} \\
g_{x} & g_{y}
\end{array}\right) \text {, }
$$

where

$$
\begin{aligned}
& f_{x}=1-\theta r\left(3 x^{2}-2 x(A+1)+A\right)-\frac{\theta x y(b x+2)}{\left(a x^{2}+b x+1\right)^{2}} \\
& f_{y}=-\frac{\theta x^{2}}{a x^{2}+b x+1} \\
& g_{x}=\frac{\theta c x y(b x+2)}{\left(a x^{2}+b x+1\right)^{2}} \\
& g_{y}=1+\theta\left(-d+\frac{c x^{2}}{a x^{2}+b x+1}\right) .
\end{aligned}
$$

The corresponding characteristic polynomial of $M_{E}$ can be written as

$$
F(\lambda)=\lambda^{2}+p \lambda+q,
$$

where $p=-f_{x}-g_{y}, q=f_{x} g_{y}-f_{y} g_{x}$.

Let $\lambda_{1}$ and $\lambda_{2}$ be two roots of equation $F(\lambda)=0$. Clearly, the local stability of the fixed point $E(x, y)$ is determined by the modules of eigenvalues $\left|\lambda_{1}\right|$ and $\left|\lambda_{2}\right|$; that is, if $\left|\lambda_{1}\right|<1$ and $\left|\lambda_{2}\right|<1$, then $E(x, y)$ is locally asymptotically stable and is termed a sink. If $\left|\lambda_{1}\right|>1$ and $\left|\lambda_{2}\right|>1$, then $E(x, y)$ is unstable and is termed a source. If $\left|\lambda_{1}\right|<1$ and $\left|\lambda_{2}\right|>1$ (or $\left|\lambda_{1}\right|>1$ and $\left.\left|\lambda_{2}\right|<1\right)$, then $E(x, y)$ is termed a saddle. If $\left|\lambda_{1}\right|=1$ or $\left|\lambda_{2}\right|=1$, then $E(x, y)$ is termed nonhyperbolic. These can be stated by the following lemma.

Lemma 2 (see [20]). Suppose $F(1)>0$; then

(i) $\left|\lambda_{1}\right|<1$ and $\left|\lambda_{2}\right|<1$ if and only if $F(-1)>0$ and $q<1$;

(ii) $\lambda_{1}=-1$ and $\left|\lambda_{2}\right| \neq 1$ if and only if $F(-1)=0$ and $p \neq 0,2$;

(iii) $\left|\lambda_{1}\right|>1$ and $\left|\lambda_{2}\right|>1$ if and only if $F(-1)>0$ and $q>1$

(iv) $\left|\lambda_{1}\right|>1$ and $\left|\lambda_{2}\right|<1$ if and only if $F(-1)<0$.

A simple computation show that two eigenvalues of $M_{E_{0}}$ are $\lambda_{1}=1-\theta r A$ and $\lambda_{2}=1-\theta d$. Also note that $F(1) \stackrel{E_{0}}{=}$ $\theta^{2} r A d>0, F(-1)=(\theta d-2)(\theta r A-2), p=\theta(A r+d)-2$, 
and $q=(\theta r A-1)(\theta d-1)$. Thus, from Lemma 2 , we have the following.

Lemma 3. The fixed point $E_{0}=(0,0)$ of model (4) always exists; moreover,

(i) $E_{0}$ is a source if $(\theta d-2)(\theta r A-2)>0, \theta r A d>d+r A$;

(ii) $E_{0}$ is a sink if $(\theta d-2)(\theta r A-2)>0, \theta r A d<d+r A$;

(iii) $E_{0}$ is a saddle if $(\theta d-2)(\theta r A-2)<0$;

(iv) $E_{0}$ is nonhyperbolic if $(\theta d-2)(\theta r A-2)=0$.

Remark 4. Define

$$
\begin{aligned}
& S_{E_{0}}^{1}=\left\{(A, a, b, c, d, \theta): \theta=\frac{2}{d}, A \neq \frac{d}{r}\right\}, \\
& S_{E_{0}}^{2}=\left\{(A, a, b, c, d, \theta): A=\frac{2}{r \theta}, \theta \neq \frac{2}{d}\right\} .
\end{aligned}
$$

From Lemma 3, we see that if $(A, a, b, c, d, \theta) \in S_{E_{0}}^{1}$ or $S_{E_{0}}^{2}$, then one of the two eigenvalues of $F(\lambda)=0$ is -1 and the other is neither 1 nor -1 , which implies that system (4) may undergo the flip bifurcation at the fixed point $E_{0}$ when parameters $(A, a, b, c, d, \theta)$ vary in a small neighborhood of $S_{E_{0}}^{1}$ or $S_{E_{0}}^{2}$.

Similarly, the eigenvalues of Jacobian $M_{E_{1}}$ are $\lambda_{1}=1-$ $r \theta(1-A)$ and $\lambda_{2}=1+\theta(c-d(a+b+1)) /(a+b+1)$. By Lemma 2 , we obtain the following results.

Lemma 5. For $E_{1}=(1,0)$, one has the following:

(i) $E_{1}$ is a sink if $0<\theta<2 / r(1-A),(a+b+1)(d-2 / \theta)<$ $c<d(a+b+1)$;

(ii) $E_{1}$ is a source if one of the following two cases holds:

$$
\begin{aligned}
\text { (1) } \theta & >2 / r(1-A), 0<c<(a+b+1)(d-2 / \theta) \text { with } \\
d & >2 / \theta \\
\text { (2) } \theta & >2 / r(1-A), c>d(a+b+1) \text {; }
\end{aligned}
$$

(iii) $E_{1}$ is a saddle if one of the following three cases holds:

$$
\begin{aligned}
& \text { (1) } 0<\theta<2 / r(1-A), 0<c<(a+b+1)(d-2 / \theta) \\
& \text { with } d>2 / \theta \text {; } \\
& \text { (2) } 0<\theta<2 / r(1-A), c>d(a+b+1) \text {; } \\
& \text { (3) } \theta>2 / r(1-A),(a+b+1)(d-2 / \theta)<c< \\
& d(a+b+1) \text {; }
\end{aligned}
$$

(iv) $E_{1}$ is nonhyperbolic if one of the following three cases holds:
(1) $\theta=2 / r(1-A)$
(2) $c=(a+b+1)(d-2 / \theta)$ with $\theta>2 / d$;
(3) $c=d(a+b+1)$.

Remark 6. Lemma 5(iv) implies that there are two parameter surfaces defined, respectively, by

$$
\begin{aligned}
& S_{E_{1}}^{1}=\left\{(A, a, b, c, d, \theta): \theta=\frac{2}{r(1-A)},\right. \\
& \left.c \neq(a+b+1)\left(d-\frac{2}{\theta}\right), c \neq d(a+b+1)\right\}, \\
& S_{E_{1}}^{2}=\left\{(A, a, b, c, d, \theta): c=(a+b+1)\left(d-\frac{2}{\theta}\right),\right. \\
& \left.\theta \neq \frac{2}{r(1-A)}, d>\frac{2}{\theta}\right\},
\end{aligned}
$$

such that one of the eigenvalues of $M_{E_{1}}$ is -1 and the other is neither 1 nor -1 when $(A, a, b, c, d, \theta) \in S_{E_{1}}^{1}$ or $S_{E_{1}}^{2}$. Thus, system (4) may undergo a flip bifurcation when $(A, a, b, c, d, \theta)$ changes in a small neighborhood of $S_{E_{1}}^{1}$ or $S_{E_{1}}^{2}$.

After calculating, we also obtain the eigenvalues of the Jacobian $M_{E_{2}}$ are $\lambda_{1}=1+\theta A r(1-A)$ and $\lambda_{2}=1+\theta(-d+$ $\left.c A^{2} /\left(a A^{2}+b A+1\right)\right)$. Note that $0<A<1$ so $\lambda_{1}>1$. Obviously, if $\left|\lambda_{2}\right|>1$, then $E_{2}$ is a source. If $\left|\lambda_{2}\right|<1$, then $E_{2}$ is a saddle. If $\left|\lambda_{2}\right|=1$, then $E_{2}$ is nonhyperbolic. By calculating, one can obtain the following.

Lemma 7. The dynamical behaviors of the fixed point $E_{2}=$ $(A, 0)$ can be stated as follows.

(i) It is a source if $c>d\left(a A^{2}+b A+1\right) / A^{2}$ or $0<c<$ $(d \theta-2)\left(a A^{2}+b A+1\right) / A^{2} \theta$ with $\theta>2 / d$.

(ii) It is a saddle if $(d \theta-2)\left(a A^{2}+b A+1\right) / A^{2} \theta<c<$ $d\left(a A^{2}+b A+1\right) / A^{2}$.

(iii) It is nonhyperbolic if $c=d\left(a A^{2}+b A+1\right) / A^{2}$ or $c=$ $(d \theta-2)\left(a A^{2}+b A+1\right) / A^{2} \theta$ with $\theta>2 / d$.

Now, we discuss local stability of the fixed point $E_{*}\left(x_{*}, y_{*}\right)$. After calculating, we get

$$
\begin{aligned}
& p=-f_{x}\left(x_{*}, y_{*}\right)-g_{y}\left(x_{*}, y_{*}\right)=-2-\theta(r C-B) ; \\
& q=1+\theta(r C-B)+d B \theta^{2},
\end{aligned}
$$

where $B=x_{*} y_{*}\left(b x_{*}+2\right) /\left(a x_{*}^{2}+b x_{*}+1\right)^{2}>0$ and $C=$ $-3 x_{*}^{2}+2(1+A) x_{*}-A$. The discriminant of equation $F(\lambda)=0$ can be calculated $\Delta=\theta^{2}\left((r C-B)^{2}-4 d B\right)$. Moreover, $F(1)=$ $d B \theta^{2}>0$ and

$$
F(-1)=d B \theta^{2}+2(r C-B) \theta+4 .
$$

Regarding $q$ and $F(-1)$ as two functions of $\theta$, then the discriminants of equations $q(\theta)=0$ and $F(-1)(\theta)=0$ are $(r C-B)^{2}-4 d B$ and $4\left((r C-B)^{2}-4 d B\right)$, respectively.

Clearly, the sign of $\Delta$ is determined by $(r C-B)^{2}-4 d B$. Therefore we have the following.

Lemma 8. Assume $d(a+b+1)<c<d\left(a+b / A+1 / A^{2}\right)$, then

(i) $E_{*}$ is a source if one of the following three cases holds:

(1) $B \leqslant r C$; 
(2) $B>r C,(r C-B)^{2} \leqslant 4 d B, \theta>(B-r C) / d B$;

(3) $B>r C,(r C-B)^{2}>4 d B, \theta>(B-r C+$ $\sqrt{\left.(B-r C)^{2}-4 d B\right)} / d B$

(ii) $E_{*}$ is a sink if one of the following two cases holds:

(1) $B>r C,(r C-B)^{2} \leqslant 4 d B, 0<\theta<(B-r C) / d B$;

(2) $B>r C,(r C-B)^{2}>4 d B, 0<\theta<(B-r C-$ $\sqrt{\left.(B-r C)^{2}-4 d B\right)} / d B$

(iii) $E_{*}$ is a saddle if $\left(B-r C-\sqrt{(B-r C)^{2}-4 d B}\right) / d B<$ $\theta<\left(B-r C+\sqrt{(B-r C)^{2}-4 d B}\right) / d B$ and $B>r C$, $(r C-B)^{2}>4 d B$

(iv) $E_{*}$ is nonhyperbolic if one of the following three cases holds:

(1) $B>r C,(r C-B)^{2} \leqslant 4 d B, \theta=(B-r C) / d B$;

(2) $B>r C,(r C-B)^{2}>4 d B, \theta=(B-r C-$ $\sqrt{\left.(B-r C)^{2}-4 d B\right)} / d B$

(3) $B>r C,(r C-B)^{2}>4 d B, \theta=(B-r C+$ $\sqrt{\left.(B-r C)^{2}-4 d B\right)} / d B$

Proof. That $F(1)>0$ always holds. Set $\lambda_{1}$ and $\lambda_{2}$ are eigenvalues of matrix $M_{E_{*}}$. We will consider two cases to prove Lemma 8.

Case 1. Assume $B \leqslant r C$, then $F(-1)>0$ and $q>1$ which yield $\left|\lambda_{1}\right|>1$ and $\left|\lambda_{2}\right|>1$.

Case 2. Assume $B>r C$. One can see that if $(r C-B)^{2} \leqslant 4 d B$, then $q \geqslant 0, F(-1) \geqslant 0$; moreover $F(-1)=0$ if and only if $(r C-B)^{2}=4 d B$ and $\theta=(B-r C) / d B$. By comparing $q$ and 1 , we obtain that if $\theta>(B-r C) / d B$, then $F(-1)>0$ and $q>1$ which lead to the fact that $E_{*}$ is a source. If $0<$ $\theta<(B-r C) / d B$ the $F(-1)>0$ and $q<1$ which lead to the fact that $E_{*}$ is a sink. If $\theta=(B-r C) / d B$ the $F(-1)=0$ and $q=1$ which lead to $\left|\lambda_{1}\right|=1$ and $\left|\lambda_{2}\right|=1$, that is, $E_{*}$ is nonhyperbolic.

In Case 2, the fact that the assumption $(r C-B)^{2}>4 d B$ leads to that the equation $F(-1)=0$ has two distinct positive roots $\theta_{1}=\left(B-r C-\sqrt{(B-r C)^{2}-4 d B}\right) / d B$ and $\theta_{2}=(B-r C+$ $\left.\sqrt{(B-r C)^{2}-4 d B}\right) / d B$ so that $F(-1)>0$ when $0<\theta<\theta_{1}$ or $\theta>\theta_{2}, F(-1)<0$ when $\theta_{1}<\theta<\theta_{2}$. Also note that $q>1$ if $\theta>(B-r C) / d B, q<1$ if $0<\theta<(B-r C) / d B$. Thus, we derive that $F(-1)>0$ and $q>1$ if $\theta>\theta_{2}, F(-1)>0$ and $q<1$ if $0<$ $\theta<\theta_{1}, F(-1)=0$ if $\theta=\theta_{1}$ or $\theta_{2}$. Following above discussion and Lemma 2, the proof of Lemma 8 is complete.

From above analysis and Lemma 8, we have two parameter surfaces:

$$
\begin{aligned}
H_{*}=\{ & (A, a, b, c, d, \theta): \theta=\frac{B-r C}{d B}, \\
& d(a+b+1)<c<d\left(a+\frac{b}{A}+\frac{1}{A^{2}}\right), \\
& 0<B-r C<2 \sqrt{d B}\} ;
\end{aligned}
$$

$$
\begin{aligned}
& S_{E_{*}}=\{(A, a, b, c, d, \theta): \theta=\theta_{1}, \\
& d(a+b+1)<c<d\left(a+\frac{b}{A}+\frac{1}{A^{2}}\right), \\
&B-r C>2 \sqrt{d B}, \theta(B-r C) \neq 2,4\} .
\end{aligned}
$$

From Lemma 8, we can see that if $(A, a, b, c, d, \theta) \epsilon$ $H_{*}$, then two eigenvalues of $M_{E_{*}}$ are a pair of conjugate complex numbers and satisfy $\left|\lambda_{1}\right|^{*}=\left|\lambda_{2}\right|=1$. Thus, when $(A, a, b, c, d, \theta)$ vary in a small neighborhood of $H_{*}$ model (4) undergoes a Neimark-Sacker bifurcation from the fixed point $E_{*}$. Similarly, if $(A, a, b, c, d, \theta) \in S_{E_{*}}$, then one of two eigenvalues of $M_{E_{*}}$ is -1 and the other is neither 1 nor -1 ; that is, when $(A, a, b, c, d, \theta)$ vary in a small neighborhood of $S_{E_{*}}$, a flip bifurcation from the fixed point $E_{*}$ can occur.

\section{Analysis of Bifurcation}

On the basis of the analysis in Section 2, by choosing parameter $\theta$ as a bifurcation parameter, we mainly investigate the flip bifurcation and Neimark-Sacker bifurcation of positive fixed point $E\left(x_{*}, y_{*}\right)$ by using the center manifold theorem and bifurcation theory of [39-41] in this section.

Giving a perturbation $\theta_{*}\left(\left|\theta_{*}\right| \ll 1\right)$ to parameter $\theta$ we consider a perturbation of model (4) as follows:

$$
\begin{aligned}
& x_{k+1}= x_{k}+\left(\theta+\theta_{*}\right) x_{k} \\
& \times\left(r\left(1-x_{k}\right)\left(x_{k}-A\right)-\frac{x_{k} y_{k}}{a x_{k}^{2}+b x_{k}+1}\right), \\
& y_{k+1}=y_{k}+\left(\theta+\theta_{*}\right) y_{k}\left(-d+\frac{c x_{k}^{2}}{a x_{k}^{2}+b x_{k}+1}\right) .
\end{aligned}
$$

Let $E(x, y)$ be a feasible fixed point of model (4). We translate $E(x, y)$ to the origin by using transformations $X_{k}=$ $x_{k}-x, Y_{k}=y_{k}-y$. Then we have (rewrite $X_{k}, Y_{k}$ as $x_{k}, y_{k}$ )

$$
\begin{aligned}
x_{k+1}= & x_{k}+\left(\theta+\theta_{*}\right)\left(x_{k}+x\right) \\
\times & \left(r\left(1-x_{k}-x\right)\left(x_{k}+x-A\right)\right. \\
& \left.-\frac{\left(x_{k}+x\right)\left(y_{k}+y\right)}{a\left(x_{k}+x\right)^{2}+b\left(x_{k}+x\right)+1}\right), \\
y_{k+1}= & y_{k}+\left(\theta+\theta_{*}\right)\left(y_{k}+y\right) \\
& \times\left(-d+\frac{c\left(x_{k}+x\right)^{2}}{a\left(x_{k}+x\right)^{2}+b\left(x_{k}+x\right)+1}\right) .
\end{aligned}
$$


Expanding model (16) as Taylor series at $\left(x_{k}, y_{k}, \theta_{*}\right)=$ $(0,0,0)$ to the second order, then we have

$$
\begin{aligned}
x_{k+1}= & a_{100} x_{k}+a_{010} y_{k}+a_{200} x_{k}^{2}+a_{110} x_{k} y_{k} \\
& +a_{101} x_{k} \theta_{*}+a_{011} y_{k} \theta_{*}+O\left(\left|x_{k}, y_{k}, \theta_{*}\right|^{4}\right) ; \\
y_{k+1}= & b_{100} x_{k}+b_{010} y_{k}+b_{200} x_{k}^{2}+b_{110} x_{k} y_{k} \\
& +b_{101} x_{k} \theta_{*}+b_{011} y_{k} \theta_{*}+O\left(\left|x_{k}, y_{k}, \theta_{*}\right|^{4}\right),
\end{aligned}
$$

where

$$
\begin{aligned}
& a_{100}=1+\theta r\left(2 x-A-3 x^{2}+2 x A\right)-\frac{\theta x y(b x+2)}{\left(a x^{2}+b x+1\right)^{2}} \\
& a_{010}=-\frac{x^{2} \theta}{a x^{2}+b x+1} ; \\
& a_{200}=\theta r(1+A-3 x)+\frac{\theta y\left(3 a x^{2}+a x^{3} b-1\right)}{\left(a x^{2}+b x+1\right)^{3}} ; \\
& a_{110}=-\frac{\theta x(b x+2)}{\left(a x^{2}+b x+1\right)^{2}} ; \\
& a_{101}=\frac{a_{100}-1}{\theta} ; \\
& b_{100}=\frac{\theta c x y(b x+2)}{\left(a x^{2}+b x+1\right)^{2}} ; \\
& b_{011}=-d \frac{x^{2}}{a x^{2}+b x+1} ; \\
& b_{010}=1+\theta\left(-d+\frac{c x^{2}}{a x^{2}+b x+1}\right) \\
& b_{110}=\frac{\theta c x(b x+2)}{\left(a x^{2}+b x+1\right)^{2}} ; \\
& b_{200}=-\frac{\theta y c\left(a b x^{3}+3 a x^{2}-1\right)}{\left(a x^{2}+b x+1\right)^{3}} ; \\
& b_{101}
\end{aligned}
$$

The flip bifurcation of model (4) at $E_{*}$ will be investigated firstly when $(A, a, b, c, d, \theta)$ varies in the small neighborhood of $S_{E_{*}}$. Similar arguments can be applied to the other cases $S_{E_{0}}^{1}, S_{E_{0}}^{2}, S_{E_{1}}^{1}, S_{E_{1}}^{2}$, and $S_{E_{2}}$.

Let $(A, a, b, c, d, \theta) \in S_{E_{*}}$ arbitrarily. From the discussion in Section 2, we can see model (4) has a unique positive fixed point $E_{*}$; its eigenvalues are $\lambda_{1}=-1$ and $-1<\lambda_{2}<0$. In this case, $x=x_{*}, y=y_{*}$, and $\theta=\theta_{1}$. Also note that $p=$ $-a_{100}-b_{010}=-a_{100}-1$ and $\lambda_{1}+\lambda_{2}=-p$ so $\lambda_{2}=2+a_{100}$. Moreover, there is a transformation

$$
\left(\begin{array}{l}
x_{k} \\
y_{k}
\end{array}\right)=\left(\begin{array}{cc}
a_{010} & a_{010} \\
-1-a_{100} & \lambda_{2}-a_{100}
\end{array}\right)\left(\begin{array}{l}
u_{k} \\
v_{k}
\end{array}\right)
$$

so that model (17) becomes

$$
\begin{aligned}
& u_{k+1}=-u_{k}+f_{1}\left(x_{k}, y_{k}, \theta_{*}\right)+O\left(\left|x_{k}, y_{k}, \theta_{*}\right|^{4}\right), \\
& v_{k+1}=\lambda_{2} v_{k}+f_{2}\left(x_{k}, y_{k}, \theta_{*}\right)+O\left(\left|x_{k}, y_{k}, \theta_{*}\right|^{4}\right),
\end{aligned}
$$

where

$$
\begin{aligned}
& f_{1}\left(x_{k}, y_{k}, \theta_{*}\right) \\
&= \frac{2 a_{200}-a_{010} b_{200}}{a_{010}\left(1+\lambda_{2}\right)} x_{k}^{2}+\frac{2 a_{110}-a_{010} b_{110}}{a_{010}\left(1+\lambda_{2}\right)} x_{k} y_{k} \\
&+\frac{2 a_{101}-a_{010} b_{101}}{a_{010}\left(1+\lambda_{2}\right)} x_{k} \theta_{*}+\frac{2 a_{011}}{a_{010}\left(1+\lambda_{2}\right)} y_{k} \theta_{*}, \\
& f_{2}\left(x_{k}, y_{k}, \theta_{*}\right) \\
&=\frac{a_{010} b_{200}-p a_{200}}{a_{010}\left(1+\lambda_{2}\right)} x_{k}^{2}+\frac{a_{010} b_{110}-p a_{110}}{a_{010}\left(1+\lambda_{2}\right)} x_{k} y_{k} \\
& \quad+\frac{a_{010} b_{101}-p a_{101}}{a_{010}\left(1+\lambda_{2}\right)} x_{k} \theta_{*}-\frac{p a_{011}}{a_{010}\left(1+\lambda_{2}\right)} y_{k} \theta_{*} .
\end{aligned}
$$

By the center manifold theorem [41], we can get the approximate representation of the center manifold $W^{c}(0,0,0)$ of model $(20)$ at the fixed point $(0,0)$ in a small neighborhood of $\theta_{1}$ as follows:

$$
\begin{aligned}
W^{c}(0,0,0)=\{ & \left(u_{k}, v_{k}, \theta_{*}\right) \in \mathbb{R}^{3}: \\
& \left.v_{k}=\eta_{0} u_{k}^{2}+\eta_{1} u_{k} \theta_{*}+O\left(\left|u_{k}, \theta_{*}\right|^{3}\right)\right\},
\end{aligned}
$$

where

$$
\begin{aligned}
& \eta_{0}=\frac{a_{010}\left(a_{010} b_{200}-p a_{200}\right)}{1-\lambda_{2}^{2}}+\frac{p\left(a_{010} b_{110}-p a_{110}\right)}{1-\lambda_{2}^{2}} ; \\
& \eta_{1}=-\frac{a_{010} b_{101}-p a_{101}}{\left(1+\lambda_{2}\right)^{2}}+\frac{p^{2} a_{011}}{a_{010}\left(1+\lambda_{2}\right)^{2}} .
\end{aligned}
$$

Furthermore, on the center manifold $W^{c}(0,0,0)$, we have

$$
\begin{aligned}
x_{k}= & a_{010}\left(u_{k}+\eta_{0} u_{k}^{2}+\eta_{1} u_{k} \theta_{*}\right)+O\left(\left|u_{k}, \theta_{*}\right|^{3}\right) ; \\
y_{k}= & p u_{k}+2\left(\eta_{0} u_{k}^{2}+\eta_{1} u_{k} \theta_{*}\right)+O\left(\left|u_{k}, \theta_{*}\right|^{3}\right) ; \\
x_{k}^{2}= & a_{010}^{2}\left(3 u_{k}^{2}+2 \eta_{0} u_{k}^{3}+2 \eta_{1} u_{k}^{2} \theta_{*}\right)+O\left(\left|u_{k}, \theta_{*}\right|^{4}\right) ; \\
x_{k} y_{k}= & -a_{010} u_{k}^{2}\left(-2 \eta_{0} u_{k}-u_{k} \eta_{0} p-p-2 \eta_{1} \theta_{*}-\eta_{1} \theta_{*} p\right) \\
& +O\left(\left|u_{k}, \theta_{*}\right|^{4}\right) ; \\
x_{k} \theta_{*}= & a_{010}\left(u_{k}+\eta_{0} u_{k}^{2}+\eta_{1} u_{k} \theta_{*}\right) \theta_{*}+O\left(\left|u_{k}, \theta_{*}\right|^{4}\right) ; \\
y_{k} \theta_{*}= & \left(p u_{k}+2 \eta_{0} u_{k}^{2}+2 \eta_{1} u_{k} \theta_{*}\right) \theta_{*}+O\left(\left|u_{k}, \theta_{*}\right|^{4}\right) .
\end{aligned}
$$

Therefore, the map $F$ which is model (4) restricted to the center manifold $W^{c}(0,0,0)$ takes the form

$$
\begin{aligned}
F\left(u_{k}\right)= & -u_{k}+h_{1} u_{k}^{2}+h_{2} u_{k} \theta_{*}+h_{3} u_{k}^{3} \\
& +h_{4} u_{k}^{2} \theta_{*}+h_{5} u_{k} \theta_{*}^{2}+O\left(\left|u_{k}, \theta_{*}\right|^{4}\right),
\end{aligned}
$$


where

$$
\begin{aligned}
h_{1}= & \frac{p\left(2 a_{110}-a_{010} b_{110}\right)}{1+\lambda_{2}}+\frac{a_{010}\left(2 a_{200}-a_{010} b_{200}\right)}{1+\lambda_{2}} ; \\
h_{2}= & \frac{2 p a_{011}}{a_{010}\left(1+\lambda_{2}\right)}+\frac{2 a_{101}-a_{010} b_{101}}{1+\lambda_{2}} ; \\
h_{3}= & \frac{2 \eta_{0}(1+p)\left(2 a_{110}-a_{010} b_{110}\right)}{1+\lambda_{2}} \\
& +\frac{2 \eta_{0} a_{010}\left(2 a_{200}-a_{010} b_{200}\right)}{1+\lambda_{2}} ; \\
h_{4}= & \frac{\eta_{1}(2+p)\left(2 a_{110}-a_{010} b_{110}\right)}{1+\lambda_{2}}+\frac{4 \eta_{0} a_{011}}{a_{010}\left(1+\lambda_{2}\right)} \\
& +\frac{2 \eta_{1} a_{010}\left(2 a_{200}-a_{010} b_{200}\right)}{1+\lambda_{2}}+\frac{\eta_{0}\left(2 a_{101}-a_{010} b_{101}\right)}{1+\lambda_{2}} ; \\
h_{5}= & \frac{4 \eta_{1} a_{011}}{a_{010}\left(1+\lambda_{2}\right)}+\frac{\eta_{1}\left(2 a_{101}-a_{010} b_{101}\right)}{1+\lambda_{2}} .
\end{aligned}
$$

In order to undergo a flip bifurcation for map (25), we require that two discriminatory quantities $\alpha_{1}$ and $\alpha_{2}$ are not zero, where

$$
\begin{aligned}
& \alpha_{1}=\left.\left(\frac{\partial^{2} F}{\partial u_{k} \partial \theta_{*}}+\frac{1}{2} \frac{\partial F}{\partial \theta_{*}} \frac{\partial^{2} F}{\partial u_{k}^{2}}\right)\right|_{(0,0)}=h_{2} \neq 0 ; \\
& \alpha_{2}=\left.\left(\frac{1}{6} \frac{\partial^{3} F}{\partial u_{k}^{3}}+\left(\frac{1}{2} \frac{\partial^{2} F}{\partial u_{k}^{2}}\right)^{2}\right)\right|_{(0,0)}=h_{3}+h_{1}^{2} .
\end{aligned}
$$

From above analysis and the theorem in [40], we have the following.

Theorem 9. If $\alpha_{2} \neq 0$, then model (4) undergoes a flip bifurcation at equilibrium $E_{*}\left(x_{*}, y_{*}\right)$ when the parameter $\theta$ varies in the small neighborhood of $\theta_{1}$. Moreover, if $\alpha_{2}>0$ (resp. $\alpha_{2}<0$ ), then the period 2 points that bifurcate from $E_{*}$ are stable (resp., unstable).

Next, we discuss the Neimark-Sacker bifurcation of $E_{*}$ when parameters $(A, a, b, c, d, \theta)$ vary in the small neighborhood of $H_{*}$. Taking $\theta=(B-r C) / d B$ (for simplicity, denoting $(B-r C) / d B$ by $\left.\theta_{0}\right)$ and choosing $\theta^{*}$ as a perturbation of bifurcation parameter, a perturbed form of model (4) is obtained as follows:

$$
\begin{aligned}
x_{k+1}= & x_{k}+\left(\theta_{0}+\theta^{*}\right) x_{k} \\
& \times\left(r\left(1-x_{k}\right)\left(x_{k}-A\right)-\frac{x_{k} y_{k}}{a x_{k}^{2}+b x_{k}+1}\right), \\
y_{k+1}=y_{k} & +\left(\theta_{0}+\theta^{*}\right) y_{k}\left(-d+\frac{c x_{k}^{2}}{a x_{k}^{2}+b x_{k}+1}\right),
\end{aligned}
$$

where $\left|\theta^{*}\right| \ll 1$ which is a small perturbation.
We transform the unique positive fixed point $E_{*}$ into the origin by using $X_{k}=x_{k}-x_{*}, Y_{k}=y_{k}-y_{*}$ :

$$
\begin{aligned}
X_{k+1}= & a_{10} X_{k}+a_{01} Y_{k}+a_{11} X_{k} Y_{k}+a_{20} X_{k}^{2} \\
& +a_{02} Y_{k}^{2}+a_{30} X_{k}^{3}+a_{21} X_{k}^{2} Y_{k}+\left(\left|X_{k}, Y_{k}\right|^{3}\right), \\
Y_{k+1}= & b_{10} X_{k}+b_{01} Y_{k}+b_{11} X_{k} Y_{k}+b_{20} X_{k}^{2} \\
& +b_{02} Y_{k}^{2}+b_{30} X_{k}^{3}+b_{21} X_{k}^{2} Y_{k}+\left(\left|X_{k}, Y_{k}\right|^{3}\right),
\end{aligned}
$$

where

$$
\begin{aligned}
& a_{10}=1+\left(\theta_{0}+\theta^{*}\right) r\left(2 x_{*}-A-3 x_{*}^{2}+2 x_{*} A\right) \\
& -\frac{\left(\theta_{0}+\theta^{*}\right) x_{*} y_{*}\left(b x_{*}+2\right)}{\left(a x_{*}^{2}+b x_{*}+1\right)^{2}} \\
& a_{01}=-\frac{d\left(\theta_{0}+\theta^{*}\right)}{c} ; \quad a_{11}=-\frac{\left(\theta_{0}+\theta^{*}\right) x_{*}\left(b x_{*}+2\right)}{\left(a x_{*}^{2}+b x_{*}+1\right)^{2}} ; \\
& a_{20}=\left(\theta_{0}+\theta^{*}\right) r\left(1+A-3 x_{*}\right) \\
& +\frac{\left(\theta_{0}+\theta^{*}\right) y_{*}\left(3 a x_{*}^{2}+a x_{*}^{3} b-1\right)}{\left(a x_{*}^{2}+b x_{*}+1\right)^{3}} \\
& a_{30}=-\frac{\left(\theta_{0}+\theta^{*}\right) y_{*}\left(a x_{*}^{2}-1\right)\left(a x_{*}^{2} b+4 a x_{*}+b\right)}{\left(a x_{*}^{2}+b x_{*}+1\right)^{4}} \\
& -\left(\theta_{0}+\theta^{*}\right) r \\
& a_{21}=\frac{\left(\theta_{0}+\theta^{*}\right)\left(a b x_{*}^{3}+3 a x_{*}^{2}-1\right)}{\left(a x_{*}^{2}+b x_{*}+1\right)^{3}} ; \\
& b_{10}=\frac{\left(\theta_{0}+\theta^{*}\right) c x_{*} y_{*}\left(b x_{*}+2\right)}{\left(a x_{*}^{2}+b x_{*}+1\right)^{2}} \text {; } \\
& b_{01}=1 ; \quad b_{20}=-\frac{\left(\theta_{0}+\theta^{*}\right) y_{*} c\left(a b x_{*}^{3}+3 a x_{*}^{2}-1\right)}{\left(a x_{*}^{2}+b x_{*}+1\right)^{3}} \text {; } \\
& b_{11}=\frac{\left(\theta_{0}+\theta^{*}\right) c x_{*}\left(b x_{*}+2\right)}{\left(a x_{*}^{2}+b x_{*}+1\right)^{2}} ; \quad b_{30}=-c a_{30} ; \\
& b_{21}=-c a_{21} \text {. }
\end{aligned}
$$

The characteristic equation associated with linearization system of model $(29)$ at $(0,0)$ is

$$
\lambda^{2}+p\left(\theta^{*}\right) \lambda+q\left(\theta^{*}\right)=0,
$$

where

$$
\begin{aligned}
& p\left(\theta^{*}\right)=-2-\left(\theta_{0}+\theta^{*}\right)(r C-B), \\
& q\left(\theta^{*}\right)=1+\left(\theta_{0}+\theta^{*}\right)(r C-B)+d B\left(\theta_{0}+\theta^{*}\right)^{2} .
\end{aligned}
$$

When parameters $(A, a, b, c, d, \theta) \in H_{*}$ and $\theta^{*}$ vary in a small neighborhood of $\theta^{*}=0$, the roots of the characteristic 
equation (31) are a pair of complex conjugate numbers $\lambda_{1}$ and $\lambda_{2}$ denoted by

$$
\begin{gathered}
\lambda_{1,2}=1+\frac{\left(\theta_{0}+\theta^{*}\right)}{2}\left(r C-B \pm i \sqrt{4 d B-(r C-B)^{2}}\right), \\
\left|\lambda_{1,2}\right|=\sqrt{q\left(\theta^{*}\right)}=\sqrt{1+\left(\theta_{0}+\theta^{*}\right)(r C-B)+d B\left(\theta_{0}+\theta^{*}\right)^{2}} .
\end{gathered}
$$

One can see that

$$
\left.\frac{d\left|\lambda_{1,2}\right|}{d \theta^{*}}\right|_{\theta^{*}=0}=\frac{B-r C}{2}>0 .
$$

On the other hand, it is required that $\lambda_{1,2}^{k} \neq 1, m=$ $1,2,3,4$ when $\theta^{*}=0$, which is equivalent to $p(0) \neq \pm 2,0,-1$. Also note that $p(0)^{2}-4 q(0)<0$ and $q(0)=1$ when $(A, a, b, c, d, \theta) \in H_{*}$. Thus, $p(0)^{2}<4$, which indicates $p(0) \neq \pm 2$. We only need to require that $p(0) \neq 0,1$; that is,

$$
(r C-B)^{2} \neq 2 d B, 3 d B
$$

which implies that $\lambda_{1,2}$ defined by (33) do not lie in the intersection of the unit circle with the coordinate axes when $\theta^{*}=0$.

Next we study the normal form of model (29) when $\theta^{*}=$ 0 .

$$
\begin{aligned}
\text { Let } \alpha & =\operatorname{Re}\left(\lambda_{1}\right), \beta=\operatorname{Im}\left(\lambda_{1}\right), \text { and } \\
T & =\left(\begin{array}{cc}
a_{01} & 0 \\
\alpha-a_{10} & -\beta
\end{array}\right) \\
& =\left(\begin{array}{cc}
a_{01} \\
-\frac{\theta_{0}(r C-B)}{2} & -\frac{\theta_{0} \sqrt{4 d B-(r C-B)^{2}}}{2}
\end{array}\right)
\end{aligned}
$$

and perform the transformations $X_{k}=a_{01} u_{k}, Y_{k}=(\alpha-$ $\left.a_{10}\right) u_{k}-\beta v_{k}$ to (29); we can obtain

$$
\begin{aligned}
& u_{k+1}=\alpha u_{k}-\beta v_{k}+G_{1}\left(u_{k}, v_{k}\right), \\
& v_{k+1}=\beta u_{k}+\alpha v_{k}+G_{2}\left(u_{k}, v_{k}\right),
\end{aligned}
$$

where

$$
\begin{aligned}
G_{1}( & \left.u_{k}, v_{k}\right) \\
= & \left(a_{20} a_{01}-\frac{a_{11}\left(a_{10}-b_{01}\right)}{2}+\frac{a_{02}\left(a_{10}-b_{01}\right)^{2}}{4 a_{01}}\right) u_{k}^{2} \\
& +\frac{a_{02} \beta^{2}}{a_{01}} v_{k}^{2}-\frac{\beta\left[a_{11} a_{01}+a_{02}\left(b_{01}-a_{10}\right)\right]}{2 a_{01}} u_{k} v_{k} \\
& +\frac{a_{01}\left[2 a_{30} a_{01}-a_{21}\left(a_{10}-b_{01}\right)\right]}{2} u_{k}^{3} \\
& -a_{01} a_{21} \beta u_{k}^{2} v_{k}+O\left(\left|u_{k}, v_{k}\right|^{4}\right) ;
\end{aligned}
$$

$$
\begin{aligned}
G_{2}\left(u_{k}, v_{k}\right) & \\
= & -\frac{1}{8 a_{01} \beta} \\
& \times\left(\left(a_{10}-b_{01}\right)\right. \\
& \times\left[a_{02}\left(a_{10}-b_{01}\right)^{2}-2 a_{01}\left(a_{11}-b_{02}\right)\left(a_{10}-b_{01}\right)\right. \\
& \left.\left.+4 a_{01}^{2}\left(a_{20}-b_{11}\right)\right]+8 b_{20} a_{01}^{3}\right) u_{k}^{2} \\
+ & \frac{1}{2 a_{01}}\left(a_{01}\left(a_{11}-2 b_{02}\right)\left(a_{10}-b_{01}\right)\right. \\
& \left.\quad-a_{02}\left(a_{10}-b_{01}\right)^{2}+2 b_{11} a_{01}^{2}\right) u_{k} v_{k} \\
- & \frac{\beta\left[2 a_{01} b_{02}+a_{02}\left(a_{10}-b_{01}\right)\right]}{2 a_{01}} v_{k}^{2} \\
- & \frac{a_{01}}{4 \beta}\left[2 a_{01}\left(a_{30}-b_{21}\right)\left(a_{10}-b_{01}\right)\right. \\
& \left.\quad-a_{21}\left(a_{10}-b_{01}\right)^{2}+4 b_{30} a_{01}^{2}\right] u_{k}^{3} \\
+ & \frac{\left[a_{21}\left(a_{10}-b_{01}\right)+2 b_{21} a_{01}\right] a_{01}}{2} u_{k}^{2} v_{k} .
\end{aligned}
$$

Let

$$
\begin{gathered}
\xi_{11}=\left.\frac{1}{4}\left[\frac{\partial^{2} G_{1}}{\partial u_{k}^{2}}+\frac{\partial^{2} G_{1}}{\partial v_{k}^{2}}+i\left(\frac{\partial^{2} G_{2}}{\partial u_{k}^{2}}-\frac{\partial^{2} G_{2}}{\partial v_{k}^{2}}\right)\right]\right|_{\left(u_{k}, v_{k}\right)=(0,0)}, \\
\xi_{20}=\frac{1}{8}\left[\frac{\partial^{2} G_{1}}{\partial u_{k}^{2}}-\frac{\partial^{2} G_{1}}{\partial v_{k}^{2}}+2 \frac{\partial^{2} G_{2}}{\partial u_{k} \partial v_{k}}\right. \\
\left.+i\left(\frac{\partial^{2} G_{2}}{\partial u_{k}^{2}}-\frac{\partial^{2} G_{2}}{\partial v_{k}^{2}}-2 \frac{\partial^{2} G_{1}}{\partial u_{k} \partial v_{k}}\right)\right]\left.\right|_{\left(u_{k}, v_{k}\right)=(0,0)}, \\
\xi_{02}=\frac{1}{8}\left[\frac{\partial^{2} G_{1}}{\partial u_{k}^{2}}-\frac{\partial^{2} G_{1}}{\partial v_{k}^{2}}-2 \frac{\partial^{2} G_{2}}{\partial u_{k} \partial v_{k}}\right. \\
\left.\quad+i\left(\frac{\partial^{2} G_{2}}{\partial u_{k}^{2}}-\frac{\partial^{2} G_{2}}{\partial v_{k}^{2}}+2 \frac{\partial^{2} G_{1}}{\partial u_{k} \partial v_{k}}\right)\right]\left.\right|_{\left(u_{k}, v_{k}\right)=(0,0)}, \\
\xi_{21}=\frac{1}{16}\left[\frac{\partial^{3} G_{1}}{\partial u_{k}^{3}}+\frac{\partial^{3} G_{1}}{\partial u_{k} \partial v_{k}^{2}}+\frac{\partial^{3} G_{2}}{\partial u_{k}^{2} \partial v_{k}}+\frac{\partial^{3} G_{2}}{\partial v_{k}^{3}}\right. \\
+i\left(\frac{\partial^{3} G_{2}}{\partial u_{k}^{3}}+\frac{\partial^{3} G_{2}}{\partial u_{k} \partial v_{k}^{2}}\right. \\
\left.\left.-\frac{\partial^{3} G_{1}}{\partial u_{k}^{2} \partial v_{k}}-\frac{\partial^{3} G_{1}}{\partial v_{k}^{3}}\right)\right]\left.\right|_{\left(u_{k}, v_{k}\right)=(0,0)} .
\end{gathered}
$$




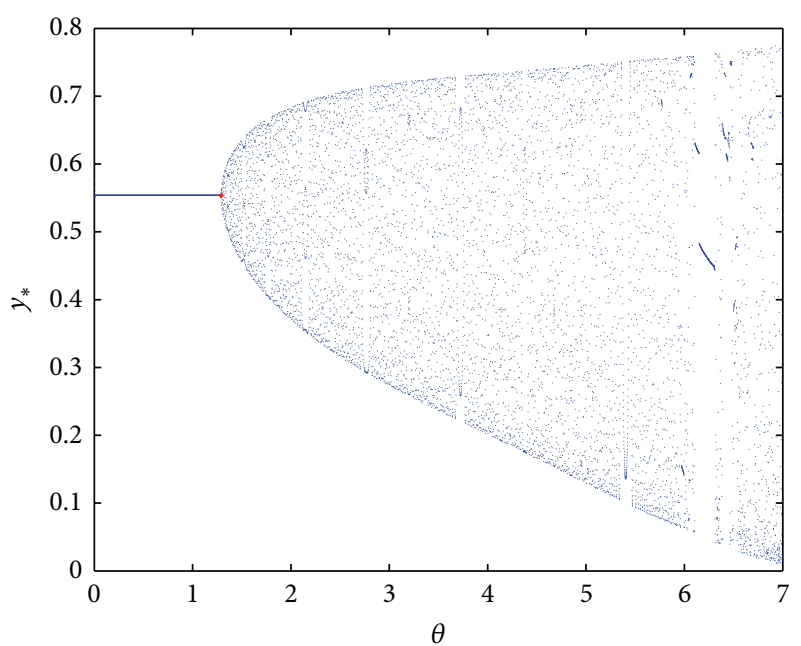

(a)

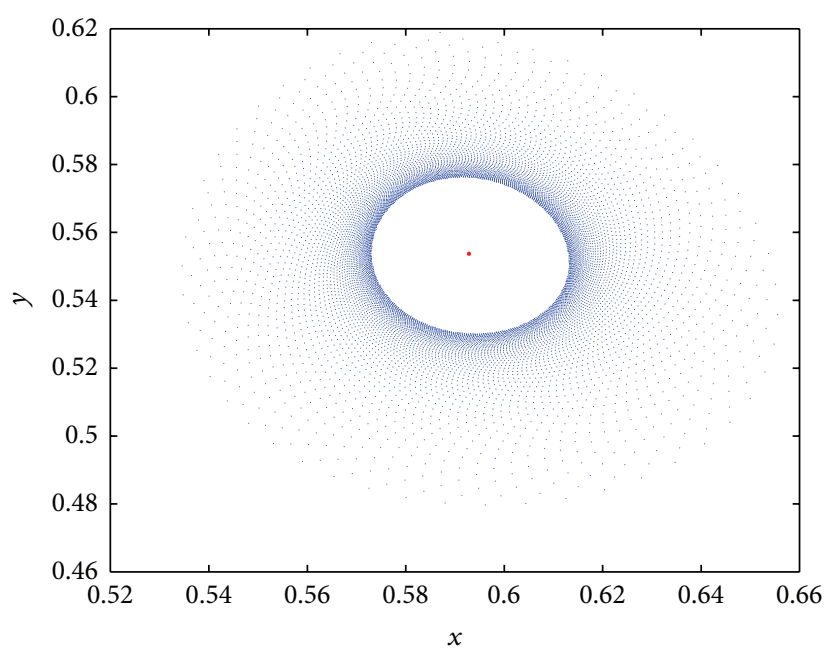

(c)

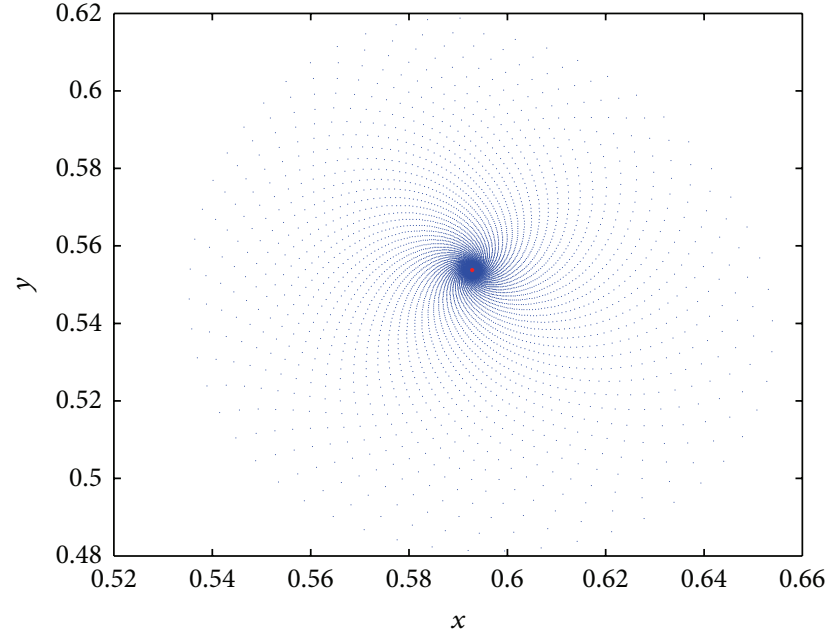

(b)

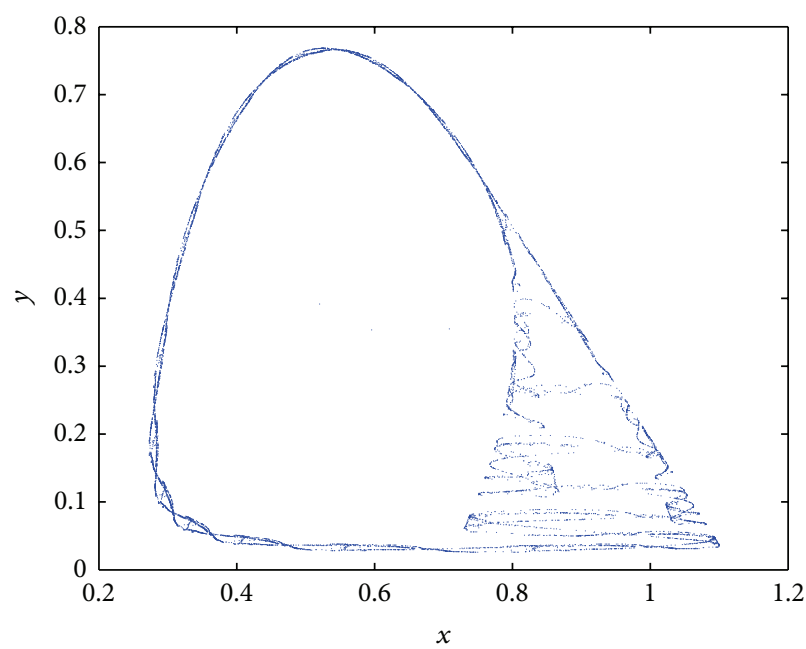

(d)

Figure 1: (a) The bifurcation diagram of system (4) with $\theta$ varying in $(0.2,2)$. The system undergoes a Neimark-Sacker bifurcation when $\theta \approx 1.290556794$ (denoted by red point). (b) A stable fixed point $E_{*}(0.5928,0.5537)$ exists when $\theta=1.2$ which occurs before the bifurcation. (c) An invariant closed curve around the fixed point $E_{*}$ created after the bifurcation which exists for $\theta=1.3$. (d) A chaotic attractor occurs when $\theta=6.6$.

By [39], we know that if the following discriminatory quantity holds, then model (38) undergoes a Neimark-Sacker bifurcation.

$$
\begin{aligned}
\mu_{*}= & -\operatorname{Re}\left(\frac{(1-2 \lambda) \bar{\lambda}^{2}}{1-\lambda} \xi_{20} \xi_{11}\right)-\frac{\left|\xi_{11}\right|^{2}}{2} \\
& -\left|\xi_{02}\right|^{2}+\operatorname{Re}\left(\bar{\lambda} \xi_{21}\right) \neq 0,
\end{aligned}
$$

where

$$
\lambda=\alpha+i \beta,
$$

$$
\begin{aligned}
& \left|\xi_{11}\right|^{2} \\
& =\left.\frac{1}{16}\left[\left(\frac{\partial^{2} G_{1}}{\partial u_{k}^{2}}+\frac{\partial^{2} G_{1}}{\partial v_{k}^{2}}\right)^{2}+\left(\frac{\partial^{2} G_{2}}{\partial u_{k}^{2}}+\frac{\partial^{2} G_{2}}{\partial v_{k}^{2}}\right)^{2}\right]\right|_{\left(u_{k}, v_{k}\right)=(0,0)},
\end{aligned}
$$

$$
\begin{aligned}
& \left|\xi_{02}\right|^{2} \\
& =\frac{1}{64}\left[\left(\frac{\partial^{2} G_{1}}{\partial u_{k}^{2}}-\frac{\partial^{2} G_{1}}{\partial v_{k}^{2}}-2 \frac{\partial^{2} G_{2}}{\partial u_{k} \partial v_{k}}\right)^{2}\right. \\
& \left.\quad+\left(\frac{\partial^{2} G_{2}}{\partial u_{k}^{2}}-\frac{\partial^{2} G_{2}}{\partial v_{k}^{2}}+2 \frac{\partial^{2} G_{1}}{\partial u_{k} \partial v_{k}}\right)^{2}\right]\left.\right|_{\left(u_{k}, v_{k}\right)=(0,0)}
\end{aligned}
$$

Form above analysis and the theorem in [39], we have the following.

Theorem 10. Let $(r C-B)^{2} \neq 2 d B, 3 d B$ and (41) hold. Then system (4) undergoes Neimark-Sacker bifurcation at the positive fixed point $E_{*}\left(x_{*}, y_{*}\right)$ when the parameter $\theta$ varies in the small neighborhood of $\theta_{0}$. Moreover, if $\mu_{*}<0$ 


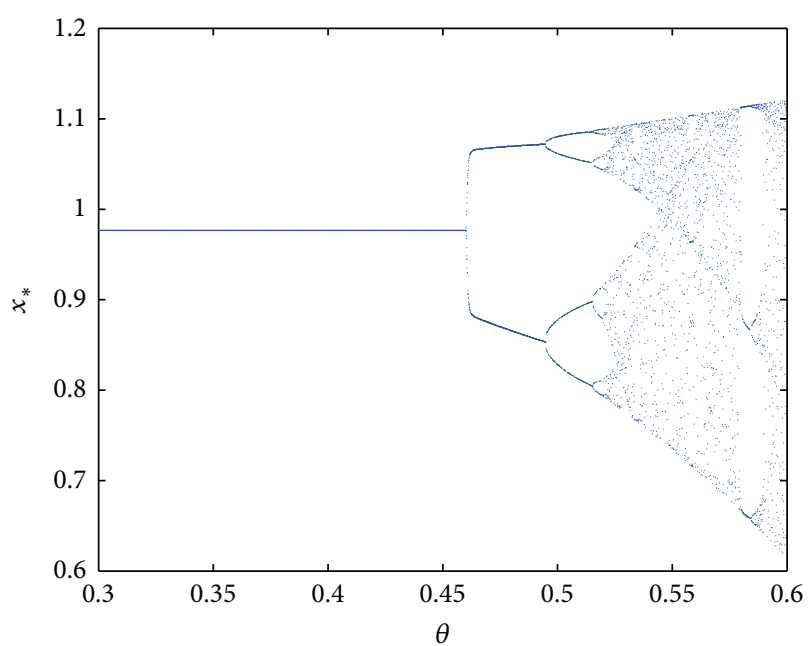

(a)

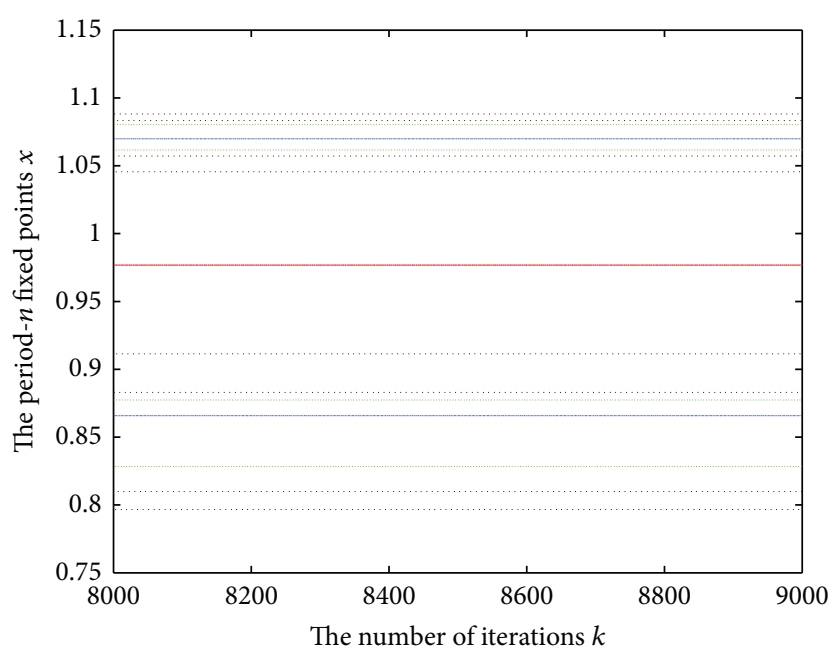

(b)

Figure 2: (a) The bifurcation diagram of system (4) with $\theta$ varying in $(0.3,0.6)$. The system undergoes a flip bifurcation when $\theta \approx 0.456$. (b) With $\theta$ increasing, system (4) undergoes period-1 point (red line corresponding to $\theta=0.36$ ), period-2 points (blue dot lines corresponding to $\theta=0.46$ ), period-4 points (green dot lines corresponding to $\theta=0.5$ ), and period- 8 points (black dot lines corresponding to $\theta=0.518$ ).

(resp., $\mu_{*}>0$ ), then an attracting (resp., repelling) invariant closed curve bifurcates from the fixed point $E_{*}$ for $\theta>\theta_{0}$ (resp., $\left.\theta<\theta_{0}\right)$.

\section{Numerical Simulations}

To confirm the above theoretical analysis, in this section we present the bifurcation diagrams and phase portraits for system (4). $\theta$ is chosen as the bifurcation parameter to verify the existence of flip bifurcation and Neimark-Sacker bifurcation.

Firstly, let $r=0.5, A=0.2, a=0.4, b=5, d=0.1$, $c=1.168$, and $\theta=1.290556794$. One can verify that all conditions in $H_{*}$ are satisfied. That is, if $\theta$ varies in a small neighborhood of $|\theta-1.290556794|<\epsilon(0<\epsilon \ll 1)$ and the other parameters are kept fixed, then system (4) undergoes a Neimark-Sacker bifurcation, which is illustrated in Figure 1.

Let $r=10, A=0.5, a=3, b=9, d=0.1$, and $c=$ 6.62. By $\theta_{1}=\left(B-r C-\sqrt{(B-r C)^{2}-4 d B}\right) / d B$, we obtain $\theta_{1} \approx 0.456$; that is, $(r, A, a, b, d, c, \theta) \in S_{E_{*}}$ when $\theta=\theta_{1}$, which leads to $\alpha_{1} \neq 0$ and $\alpha_{2}>0$. Moreover, $E_{*}$ is stable when $\theta<\theta_{1}$ and loses its stability when $\theta$ reaches and passes $\theta_{1}$, which leads to a cascade of the period doubling with $\theta$ increasing (see Figure 2).

\section{Conclusion}

In this paper, a discrete-time prey-predator model with generalized Holling type III functional response and Allee effect has been presented. The stability of fixed points and the existence of flip bifurcation and Neimark-Sacker bifurcation are investigated. Our analysis shows that the strong Allee effect can change topological structure and dynamics of the system. The combined influence of Allee effect and the other parameters induce system (4) to yield more rich dynamical behaviors including chaotic bands and chaotic attractors which do not exist in the continuous case. These results reported in this paper could be very useful for the biologists who devote to study the discrete-time prey-predator models.

\section{Conflict of Interests}

The authors declare that there is no conflict of interests regarding the publication of this paper.

\section{Acknowledgments}

This paper is supported by NSFC (11226142), Foundation of Henan Educational Committee (2012A110012), Foundation of Henan Normal University (2011QK04, 2012PL03), and Scientific Research Foundation for Ph.D. of Henan Normal University (no. 1001).

\section{References}

[1] J. Beddington, "Mutual interference between parasites or predators and its effect on searching efficiency," Journal of Animal Ecology, vol. 44, pp. 331-340, 1975.

[2] D. DeAngelis, R. Goldstein, and R. V. O’Neill, "A model for tropic interaction," Ecology, vol. 56, no. 4, pp. 881-892, 1975.

[3] P. Crowley and E. Martin, "Functional responses and interference within and between year classes of a dragonfly population," Journal of the North American Benthological Society, vol. 8, pp. 211-221, 1989.

[4] C. S. Holling, "The functional response of predator to prey density and its role in mimicry and population regulation," Memoirs of the Entomological Society of Canada, vol. 45, pp. 160, 1965.

[5] M. Sambath and K. Balachandran, "Spatiotemporal dynamics of a predator-prey model incorporating a prey refuge," The Journal 
of Applied Analysis and Computation, vol. 3, no. 1, pp. 71-80, 2013.

[6] X. Liu and M. Han, "Chaos and Hopf bifurcation analysis for a two species predator-prey system with prey refuge and diffusion," Nonlinear Analysis: Real World Applications, vol. 12, no. 2, pp. 1047-1061, 2011.

[7] X. Liu, Y. Liu, and J. Wang, "Bogdanov-Takens bifurcation of a delayed ratio-dependent Hollig-TANner predator prey system," Abstract and Applied Analysis, vol. 2013, Article ID 898015, 5 pages, 2013.

[8] Y. Lamontagne, C. Coutu, and C. Rousseau, "Bifurcation analysis of a predator-prey system with generalised Holling type III functional response," Journal of Dynamics and Differential Equations, vol. 20, no. 3, pp. 535-571, 2008.

[9] S.-R. Zhou, Y.-F. Liu, and G. Wang, "The stability of predatorprey systems subject to the Allee effects," Theoretical Population Biology, vol. 67, no. 1, pp. 23-31, 2005.

[10] D. Hadjiavgousti and S. Ichtiaroglou, "Allee effect in a preypredator system," Chaos, Solitons and Fractals, vol. 36, no. 2, pp. 334-342, 2008.

[11] X. Zhang, Q.-L. Zhang, and Z. Xiang, "Bifurcation analysis of a singular bioeconomic model with Allee effect and two time delays," Abstract and Applied Analysis, vol. 2014, Article ID 745296, 12 pages, 2014.

[12] G. A. van Voorn, L. Hemerik, M. P. Boer, and B. W. Kooi, "Heteroclinic orbits indicate overexploitation in predator-prey systems with a strong Allee effect," Mathematical Biosciences, vol. 209, no. 2, pp. 451-469, 2007.

[13] M. S. Fowler and G. D. Ruxton, "Population dynamic consequences of Allee effects," Journal of Theoretical Biology, vol. 215, no. 1, pp. 39-46, 2002.

[14] M. A. McCarthy, "The Allee effect, finding mates and theoretical models," Ecological Modelling, vol. 103, no. 1, pp. 99-102, 1997.

[15] C. E. Brassil, "Mean time to extinction of a metapopulation with an Allee effect," Ecological Modelling, vol. 143, no. 1-2, pp. 9-16, 2001.

[16] F. Courchamp, L. Berec, and J. Gascoigne, Allee Effects in Ecology and Conservation, Oxford Biology, Oxford University Press, New York, NY, USA, 2009.

[17] B. Dennis, "Allee effects: population growth, critical density, and the chance of extinction," Natural Resource Modeling, vol. 3, no. 4, pp. 481-538, 1989.

[18] P. Aguirre, E. González-Olivares, and E. Sáez, “Two limit cycles in a Leslie-Gower predator-prey model with additive Allee effect," Nonlinear Analysis: Real World Applications, vol. 10, no. 3, pp. 1401-1416, 2009.

[19] R. López-Ruiz and D. Fournier-Prunaret, "Indirect Allee effect, bistability and chaotic oscillations in a predator-prey discrete model of logistic type," Chaos, Solitons and Fractals, vol. 24, no. 1, pp. 85-101, 2005.

[20] C. Celik and O. Duman, "Allee effect in a discrete-time predatorprey system," Chaos, Solitons \& Fractals, vol. 40, no. 4, pp. 19561962, 2009.

[21] H. Merdan and O. Duman, "On the stability analysis of a general discrete-time population model involving predation and Allee effects," Chaos, Solitons \& Fractals, vol. 40, no. 3, pp. 1169-1175, 2009.

[22] H. T. M. Eskola and P. Kalle, "On the mechanistic underpinning of discrete-time population models with Allee effect," Theoretical Population Biology, vol. 72, no. 1, pp. 41-51, 2007.
[23] W.-X. Wang, Y.-B. Zhang, and C.-Z. Liu, "Analysis of a discretetime predator-prey system with Allee effect," Ecological Complexity, vol. 8, no. 1, pp. 81-85, 2011.

[24] I. Djellit, M. L. Sahari, and A. Hachemi, "Complex dynamics in 2-species predator-prey systems," The Journal of Applied Analysis and Computation, vol. 3, no. 1, pp. 11-20, 2013.

[25] Z. Jing and J. Yang, "Bifurcation and chaos in discrete-time predator-prey system," Chaos, Solitons and Fractals, vol. 27, no. 1, pp. 259-277, 2006.

[26] X. Liu and D. Xiao, "Complex dynamic behaviors of a discretetime predator-prey system," Chaos, Solitons \& Fractals, vol. 32, no. 1, pp. 80-94, 2007.

[27] X. Zhang, Q.-1. Zhang, and V. Sreeram, "Bifurcation analysis and control of a discrete harvested prey-predator system with Beddington-DeAngelis functional response," Journal of the Franklin Institute, vol. 347, no. 7, pp. 1076-1096, 2010.

[28] H. N. Agiza, E. M. ELabbasy, H. EL-Metwally, and A. A. Elsadany, "Chaotic dynamics of a discrete prey-predator model with Holling type II," Nonlinear Analysis: Real World Applications, vol. 10, no. 1, pp. 116-129, 2009.

[29] Z. He and X. Lai, "Bifurcation and chaotic behavior of a discretetime predator-prey system," Nonlinear Analysis: Real World Applications, vol. 12, no. 1, pp. 403-417, 2011.

[30] D. Blackmore, J. Chen, J. Perez, and M. Savescu, "Dynamical properties of discrete Lotka-Volterra equations," Chaos, Solitons \& Fractals, vol. 12, no. 13, pp. 2553-2568, 2001.

[31] D. Summers, J. G. Cranford, and B. P. Healey, "Chaos in periodically forced discrete-time ecosystem models," Chaos, Solitons \& Fractals, vol. 11, no. 14, pp. 2331-2342, 2000.

[32] X. Liao, S. Zhou, and Z. Ouyang, "On a stoichiometric two predators on one prey discrete model," Applied Mathematics Letters, vol. 20, no. 3, pp. 272-278, 2007.

[33] W. Yang and X. Li, "Permanence for a delayed discrete ratiodependent predator-prey model with monotonic functional responses," Nonlinear Analysis: Real World Applications, vol. 10, no. 2, pp. 1068-1072, 2009.

[34] X. T. Yang, "Uniform persistence and periodic solutions for a discrete predator-prey system with delays," Journal of Mathematical Analysis and Applications, vol. 316, no. 1, pp. 161-177, 2006.

[35] X. Yang, Y. Liu, and J. Chen, "Uniform persistence for a discrete predator-prey system with delays," Applied Mathematics and Computation, vol. 218, no. 4, pp. 1174-1179, 2011.

[36] F. Chen, "Permanence and global attractivity of a discrete multispecies Lotka-Volterra competition predator-prey systems," Applied Mathematics and Computation, vol. 182, no. 1, pp. 3-12, 2006.

[37] X. Chen, "Periodicity in a nonlinear discrete predator-prey system with state dependent delays," Nonlinear Analysis: Real World Applications, vol. 8, no. 2, pp. 435-446, 2007.

[38] Y. Xia, J. Cao, and M. Lin, "Discrete-time analogues of predatorprey models with monotonic or nonmonotonic functional responses," Nonlinear Analysis: Real World Applications, vol. 8, no. 4, pp. 1079-1095, 2007.

[39] J. Guckenheimer and P. Holmes, Nonlinear Oscillations Dynamical Systems and Bifurcations of Vector Fields, Springer, New York, NY, USA, 1983.

[40] C. Robinson, Dynamical Systems: Stability, Symbolic Dynamics and Chaos, CRC Press, Boca Raton, Fla, USA, 2nd edition, 1999.

[41] J. Carr, Application of Center Manifold Theory, Springer, New York, NY, USA, 1981. 


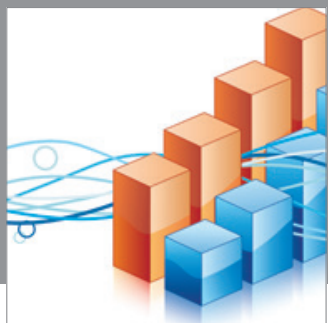

Advances in

Operations Research

mansans

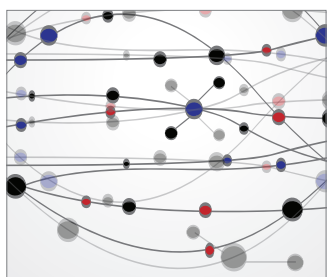

The Scientific World Journal
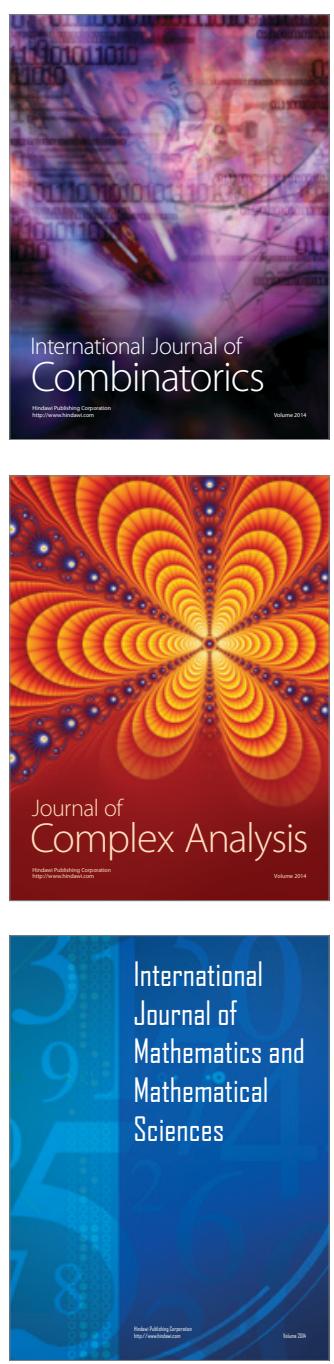
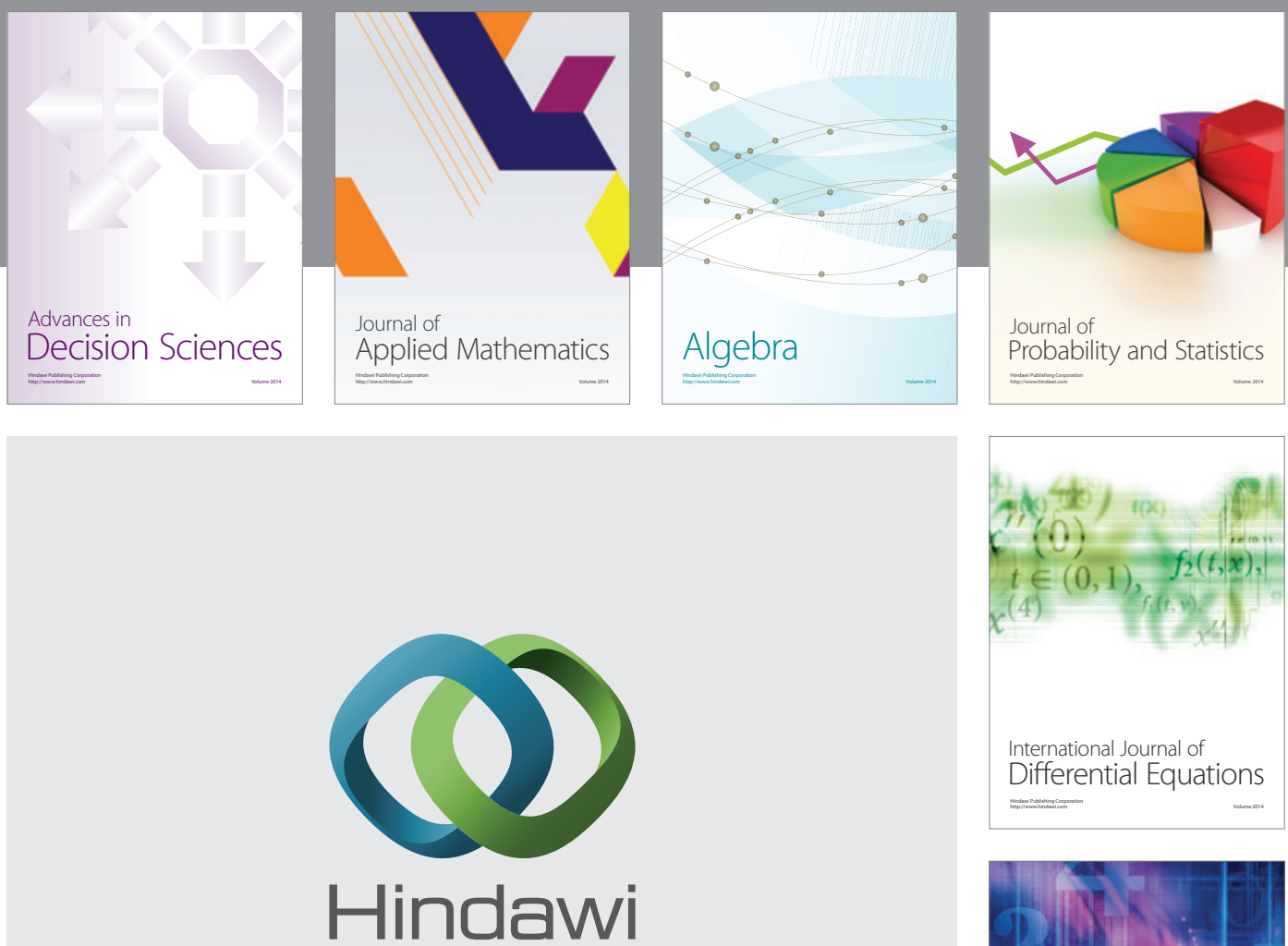

Submit your manuscripts at http://www.hindawi.com
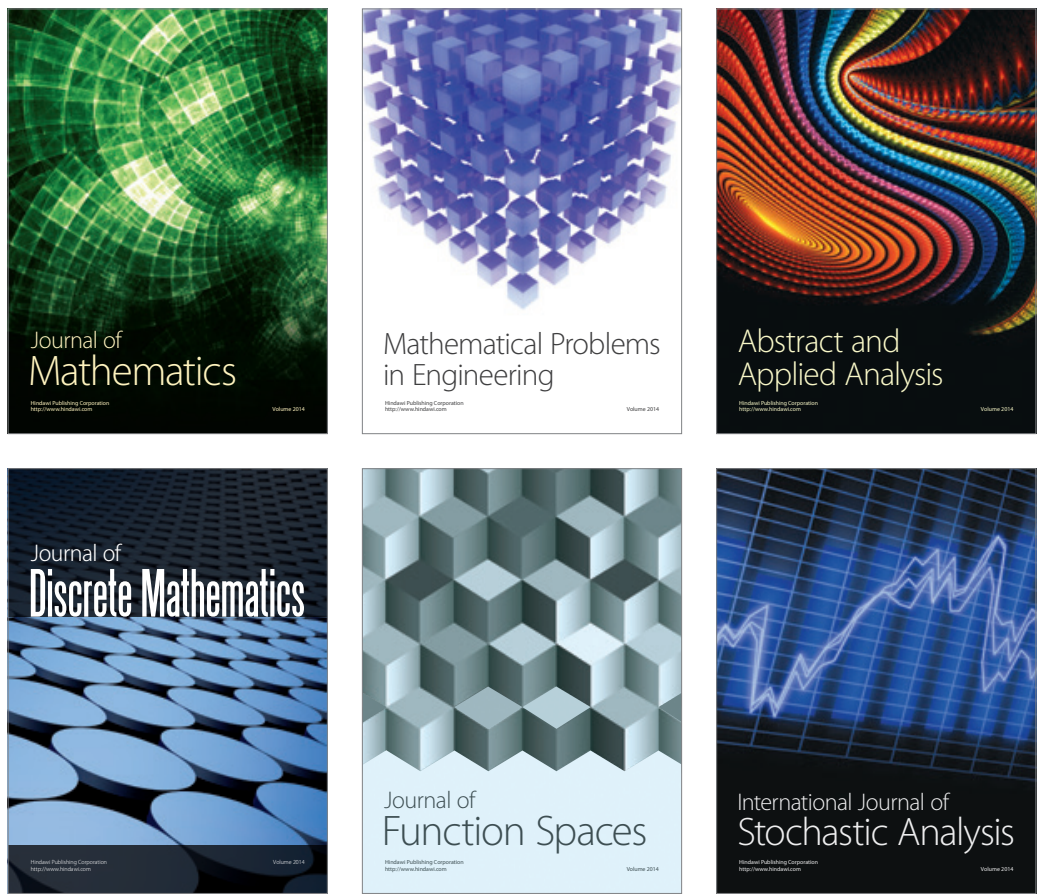

Journal of

Function Spaces

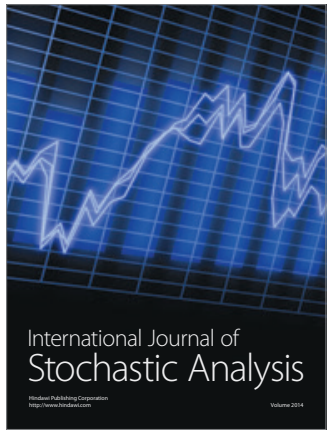

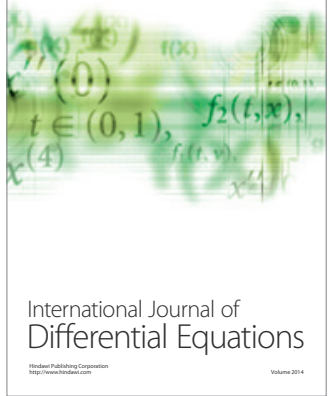
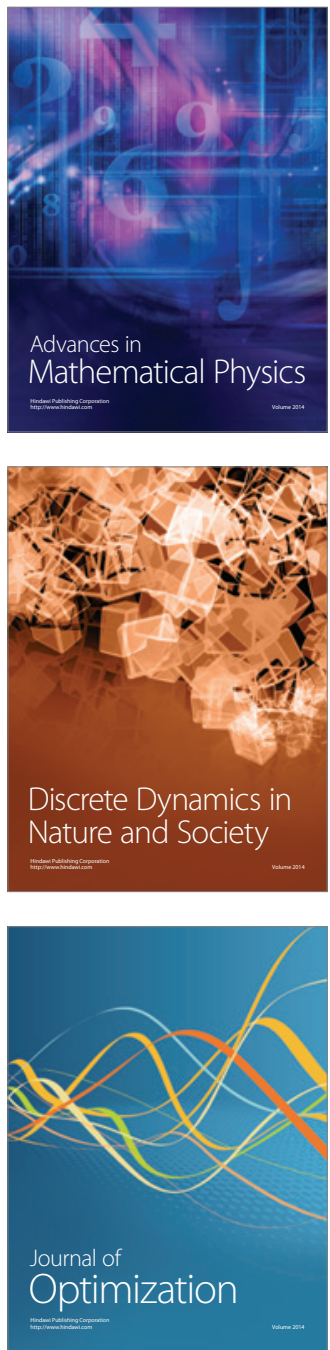